\title{
Undertriage in trauma: an ignored quality indicator?
}

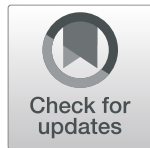

Elisabeth Jeppesen ${ }^{1,2^{*}}$ (D), Mathias Cuevas- $\varnothing_{\text {strem }}{ }^{1,2}$, Cathrine Gram-Knutsen ${ }^{2}$ and Oddvar Uleberg ${ }^{1,3,4}$

\begin{abstract}
Background: Early identification of life-threatening injuries is essential to reduce morbidity and mortality in trauma patients. Failure to detect severe injury may cause delayed diagnosis and therapeutic interventions and is associated with increased morbidity. A national trauma system will contribute to ensure the optimal care for seriously injured patients throughout the treatment chain by, among other things, defining a sensitive triage tool for identifying severe injury and contribute to correct treatment destination. In 2017, a National trauma plan was implemented in Norway and several quality indicators were recommended to ensure an evaluation of potential gaps between achieved and desired quality, and thereby highlighting areas with potential for quality improvement. With this commentary, we want to draw attention to, what we believe is, an ignoring of an important quality indicator: undertriage in trauma.

Main body: Severely injured patients not met by a trauma team is commonly referred to as undertriage. An undertriage rate below $5 \%$ is an internationally recognized quality indicator in trauma care and is emphasized in the Norwegian national trauma plan. However, whether hospitals measure and report data about undertriage, have received little attention. Therefore, a national survey was performed among Norwegian hospitals, where thirty-seven of forty trauma receiving hospitals contributed. The results of the survey showed that only half of Norwegian trauma hospitals were capable of providing these data. The results of this survey show that currently the national trauma system is not equipped to obtain important data on an important and specific quality indicator. An ongoing discussion at a national level is how to define severe injury, which may alter future definitions on undertriage.

Conclusions: Knowledge of undertriage in trauma is important to enhance patient safety, increase the precision of the triage tool and provide valuable learning information to individual hospitals and prehospital services. Currently only half of Norwegian hospitals who receive trauma patients report undertriage rates and unfortunately, only few hospital administrators request these data.
\end{abstract}

Keywords: Trauma system, Undertriage, Quality indicator

\footnotetext{
* Correspondence: elisabeth.jeppesen@norskluftambulanse.no

${ }^{1}$ Department of Research and Development, Norwegian Air Ambulance

Foundation, NO-0103 Oslo, Norway

${ }^{2}$ Faculty of Health Science, University of Stavanger, Stavanger, Norway

Full list of author information is available at the end of the article
}

(C) The Author(s). 2020 Open Access This article is licensed under a Creative Commons Attribution 4.0 International License, which permits use, sharing, adaptation, distribution and reproduction in any medium or format, as long as you give appropriate credit to the original author(s) and the source, provide a link to the Creative Commons licence, and indicate if changes were made. The images or other third party material in this article are included in the article's Creative Commons licence, unless indicated otherwise in a credit line to the material. If material is not included in the article's Creative Commons licence and your intended use is not permitted by statutory regulation or exceeds the permitted use, you will need to obtain permission directly from the copyright holder. To view a copy of this licence, visit http://creativecommons.org/licenses/by/4.0/. The Creative Commons Public Domain Dedication waiver (http://creativecommons.org/publicdomain/zero/1.0/) applies to the data made available in this article, unless otherwise stated in a credit line to the data. 


\section{Background}

Early identification of life-threatening injuries is essential to reduce morbidity and mortality [1]. Triage is used at the scene for multiple purposes; to separate patients with potentially serious injuries from those with minor injuries, prioritizing patients for adequate transport, choosing the right destination and finally choosing the correct response at the receiving hospital. Sensitive triage tools which include physiologic variables, specific anatomic injuries, mechanism of injuries and certain special causes (e.g. age and severe co-morbidity) assist prehospital personnel in the triage process [1]. Suspicion that a person is seriously injured is based on all available knowledge - but due to the need of rapid transportation and treatment, the decision to consider the patient as seriously injured is often based on limited information. Patients who are severely injured (Injury Severity Score [ISS] greater than 15) and not received by a trauma team are commonly referred to as undertriaged (false-negatives) $[2,3]$. Failure to detect severe injury may cause delayed diagnosis and therapeutic interventions and is associated with increased morbidity [4, 5]. Conversely, considering a patient to be severely injured where there appears to be less severe injury (ISS $<15$ ), is defined as overtriage. The overtriage rate is defined to capture the proportion of unnecessary use of hospital resources on minor trauma (resource overutilization) and could create concurrent conflicts for treating health care professionals with adverse consequences for other patients [6].

An undertriage rate of $<5 \%$ is often considered as acceptable according to the American College of Surgeons Committee on Trauma (ACS-COT) [1]. However, previous Norwegian studies have shown substantial higher rates of undertriage at Norwegian hospitals, varying between 13 and 28\% [7-9], with similar rates seen in systems in the Netherlands and the United States [10, 11]. Therefore, undertriage in trauma is still a challenge even for the most highly developed trauma systems [12].

A trauma system should help to ensure that seriously injured patients are optimally cared for throughout the treatment chain [1]. In 2017, a National trauma plan was implemented in Norway to ensure an optimal systematic approach and common practices to improve trauma management throughout the country [13]. Several quality indicators were recommended to ensure an evaluation of potential gaps between achieved and desired quality, and thereby highlighting areas with potential for quality improvement. One of the most important quality indicators was the rate of undertriage at Norwegian hospitals. An undertriage rate $<5 \%$ was seen as an important indicator of triage precision. However, whether hospitals themselves actually are capable and have the capacity to report these and other quality indicators, have received all too little attention. With this commentary, we want to draw attention to, what we believe is, an ignoring of an important quality indicator: undertriage in trauma, defined as patients with ISS $>15$ and not received by a trauma team.

\section{Main text}

A national survey was performed where a questionnaire was sent to certified registrars in all hospitals reporting data to the Norwegian Trauma registry (NTR) in 2017. NTR should include all patients with New Injury Severity Score $[\mathrm{NISS}]>12$, head injury AIS $\geq 3$ and patients with penetrating injuries proximal to elbow or knee, regardless of whether they have received by a trauma team or not. The registrars were asked if they identify and include patients without trauma team activation in the registry. Thirty-seven of forty hospitals, including all four trauma centres, responded to the survey. Nineteen hospitals (51\%) stated that they identified and reported undertriage rates. According to results from open questionnaires in the survey, lack of time and resources (57\%) were identified as the main barrier to not be able to identify undertriage. Secondly, the working process of retrieving these data was labour-intensive as no precise and adequate technical solutions were available for the data extraction. In addition, half of the hospitals (19 of 37) stated that data on undertriage was not requested, or of interest, by their own hospital administration. The results of the survey shown that only half of Norwegian trauma hospitals were capable of providing data on a nationally defined quality indicator on trauma management.

A study by Wisborg et al., found that only $50 \%$ of seriously injured patients in Norway are offered advanced anaesthesiology-led care outside hospitals. The combined effect of both pre- and in-hospital undertriage may lead to negative patient outcome [14]. In a study by Voskens et al., the undertriage rate among older adults were $38.6 \%$ [10]. This result highlights the specific challenges of increased undertriage seen in the older population. Experiences from clinical practice show that undertriaged geriatric patients with low-energy trauma can have few presenting complaints and clinical findings despite severe injuries, typically in the head or as occult internal bleedings. In the absence of red flags, the acuity is low, and they are often evaluated in a community casualty clinic before presenting to hospital. As the current triage algorithms were primarily developed based on a younger trauma population, the increased cohort of older trauma patients with subtle injuries underlines the increased need attention on undertriage rates [12].

Throughout many years the ability of field triage criteria to identify precisely severely injured patients have been addressed [2]. The results of this survey show that currently the national trauma system is not equipped to 
obtain important data on an important and specific quality indicator. An ongoing discussion at a national level is how to define severe injury, which may alter future definitions on undertriage. Today, the most commonly used indicator of severity is the ISS, but variables such as the New Injury Severity Score (NISS), length of hospital stay and performed hospital interventions have also been proposed as measures of severity [15]. Though irrespective on how we define severity, the ability to measure and provide a follow-up on an established quality indicator is imperative for improvement initiatives. A lack of follow-up may lead to the disregard of undertriage as a quality indicator.

\section{Conclusions}

Knowledge of undertriage in trauma is important to enhance patient safety, increase the precision of the triage tool and provide valuable learning information to individual hospitals and prehospital services. Currently, only half of Norwegian hospitals who receive trauma patients report undertriage rates and unfortunately, only a few hospital administrators request these data. Lack of time, resources and adequate technical solutions are the main challenges in order not to prioritize this task.

\section{Abbreviations}

ISS: Injury Severity Score; NISS: New Injury Severity Score; NTR: Norwegian Trauma Registry

\section{Acknowledgements}

Not applicable.

\section{Authors' contributions}

CGK conducted the undertriage study and EJ contributed to the method. CGK, OU and MC $\varnothing$ contributed to the analysis and drafting. EJ, MCØ and OU were the major contributors in writing the manuscript. All authors read and approved the final manuscript.

\section{Authors' information \\ EJ is an associate professor at the University of Stavanger (UIS), in the Pre- Hospital Critical Care master program (PHCC). She also works as a senior re- searcher at the Norwegian Air Ambulance Foundation (NAAF). MCØ is a medical doctor and a PhD-student at the UIS and NAAF. CGK work as a Para- medic and has completed the master program PHCC at the UIS. OU is an consultant anesthetist at St. Olav's University Hospital in Trondheim and an associate professor at the Norwegian University of Science and Technology (NTNU). He also works as a senior researcher at the Norwegian Air Ambu- lance Foundation (NAAF).}

\section{Funding}

The authors received no external funding.

\section{Availability of data and materials}

The datasets used during the current study are available from the corresponding author on reasonable request.

\section{Ethics approval and consent to participate}

The study used anonymized patient data, collected by local and national approved health registries according to The Norwegian Health Research Act. The regional ethical committee (Reference number: REC Central Norway 2016/2169) categorized this as a quality improvement project. The local health trusts gave institutional approval.
Consent for publication

Not applicable.

\section{Competing interests}

The authors declare that they have no competing interests or disclosures.

\section{Author details}

'Department of Research and Development, Norwegian Air Ambulance Foundation, NO-0103 Oslo, Norway. ${ }^{2}$ Faculty of Health Science, University of Stavanger, Stavanger, Norway. ${ }^{3}$ Department of Emergency Medicine and Pre-Hospital Services, St. Olav's University Hospital, NO-7006 Trondheim, Norway. ${ }^{4}$ Department of Circulation and Medical Imaging, Norwegian University of Science and Technology, NO-7006 Trondheim, Norway.

Received: 13 February 2020 Accepted: 27 April 2020

Published online: 06 May 2020

References

1. American College of Surgeons Committee on Trauma. Resources for Optimal Care og the Injured Patient: 2014, 6th. ed. Chicago: American College of Surgeons; 2014.

2. Vinjevoll OP, Uleberg O, Cole E. Evaluating the ability of a trauma team activation tool to identify severe injury: a multicentre cohort study. Scand J Trauma Resusc Emerg Med. 2018;26:63. https://doi.org/10.1186/s13049-0180533-y.

3. Rehn M, Perel P, Blackhall K, Lossius HM. Prognostic models for the early care of trauma patients: a systematic review. Scand J Trauma Resusc Emerg Med. 2011;19:17. https://doi.org/10.1186/1757-7241-19-17.

4. Mackenzie EJ, Rivara FP, Jurkovich GJ, Nathens AB, Frey KP, Egleston BL, et al. A National Evaluation of the effect of trauma-center care on mortality. N Engl J Med. 2006;354:366-78.

5. van Rein EAJ, Houwert RM, Gunning AC, Lichtveld R, Leenen LPH, van Heijl $M$. Accuracy of prehospital triage protocols in selecting major trauma patients: a systematic review. J Trauma Acute Care Surg. 2017. https://doi. org/10.1097/TA.0000000000001516.

6. Fishman PE, Shofer FS, Robey JL, et al. The impact of trauma activations on the care of emergency department patients with potential acute coronary syndromes. Ann Emerg Med. 2006;48:347.

7. Uleberg, Vinjevoll, Eriksson, Aadahl, Skogvoll. Overtriage in trauma what are the causes? Acta Anaesthesiol Scand. 2007;51:1178-83.

8. Dehli T, Fredriksen K, Osbakk SA, Bartnes K. Evaluation of a university hospital trauma team activation protocol. Scand J Trauma Resusc Emerg Med. 2011;19:18. https://doi.org/10.1186/1757-7241-19-18.

9. Dehli T, Monsen SA, Fredriksen K, Bartnes K. Evaluation of a trauma team activation protocol revision: a prospective cohort study. Scand J Trauma Resusc Emerg Med. 2016;24:105. https://doi.org/10.1186/s13049-016-0295-3.

10. Voskens FJ, van Rein EAJ, van der Sluijs R, Houwert RM, Lichtveld RA, Verleisdonk EJ, et al. Accuracy of Prehospital triage in selecting severely injured trauma patients. JAMA Surg. 2018;153:322-7. https://doi.org/10.1001/ jamasurg.2017.4472.

11. Kodadek LM, Selvarajah S, Velopulos CG, Haut ER, Haider AH. Undertriage of older trauma patients: is this a national phenomenon? I Surg Res. 2015;199: 220-9. https://doi.org/10.1016/j.jss.2015.05.017.

12. Newgard CD, Uribe-Leitz T, Haider AH. Undertriage remains a vexing problem for even the Most highly developed trauma systems: the need for innovations in field triage. JAMA Surg. 2018;153:328. https://doi.org/10.1001/ jamasurg.2017.4499.

13. Norwegian National Trauma Plan [https://traumeplan.no/] Accessed 5 Oct 2020

14. Wisborg T, Ellensen EN, Svege I, Dehli T. Are severely injured trauma victims in Norway offered advanced pre-hospital care? National, retrospective observational cohort. Acta Anaesthesiol Scand. 2017;61:841-7. https://doi. org/10.1111/aas.12931.

15. Paffrath T, Lefering R, Flohe $S$, TraumaRegister DGU. How to define severely injured patients? - an injury severity score (ISS) based approach alone is not sufficient. Injury. 2014;45(Suppl 3):S64-9.

\section{Publisher's Note}

Springer Nature remains neutral with regard to jurisdictional claims in published maps and institutional affiliations. 